Voix et Images

\title{
Sans le ciel ni le mondain : pour retrouver le monde
}

\section{Luc Bonenfant}

Volume 34, numéro 3 (102), printemps-été 2009

Trajectoires de l'auteur dans le théâtre contemporain

URI : https://id.erudit.org/iderudit/037672ar

DOI : https://doi.org/10.7202/037672ar

Aller au sommaire du numéro

\section{Éditeur(s)}

Université du Québec à Montréal

ISSN

0318-9201 (imprimé)

1705-933X (numérique)

Découvrir la revue

Citer ce compte rendu

Bonenfant, L. (2009). Compte rendu de [Sans le ciel ni le mondain : pour retrouver le monde]. Voix et Images, 34(3), 141-146.

https://doi.org/10.7202/037672ar d'utilisation que vous pouvez consulter en ligne.

https://apropos.erudit.org/fr/usagers/politique-dutilisation/ 


\section{P O É S I E}

Sans le ciel ni le mondain: pour retrouver le monde

$++$

LUC BONENFANT

Université du Québec à Montréal

La douleur traverse la poésie actuelle comme un cliché lancinant. Quand il est torturé, souffrant ou affligé - ce qui ne revient pas au même -, le poète contemporain qui adopte une posture lyrique fait souvent part de ses états d'âme sans autre médiation que celle de la tonalité toute personnelle de sa douleur immédiate. Parfois, il arrive néanmoins à formaliser cette douleur dans un langage où elle réussit heureusement à dépasser la stricte énonciation ontologique du cri primaire. Si les tons respectifs de leurs voix m'apparaissent opposés tant l'un semble placide et l'autre rugissante, Philippe More et Catherine Morency appartiennent à cette race de poètes lyriques chez qui le travail énonciatif et esthétique du vers ouvre à la capacité réelle du poète de se transformer en sujet lyrique. Le lyrisme apparaît chez eux comme la construction politique d'une parole où le sujet s'entend bel et bien comme un procès ${ }^{1}$ qui ne laisse pas de souligner sa situation discursive particulière.

Brouillons pour un siècle abstrait ${ }^{2}$, de Philippe More, forme le dernier volet d'une espèce de triptyque amorcé par Théâtre de l'apesanteur, en 2006, et continué par Nos images d'aphasie, en 2007. Avec ces recueils, le poète nous avait appris une exigence qu'il confirme ici puisqu' on "s'habille comme on se quitte soi-même/ comme on s'enrôle corps après corps/[...] en cédant sa propre voix à sa doublure » (56). Naguère portée par le sujet, l'aphasie est désormais projetée depuis lui. De ce point de vue, Brouillons pour un siècle abstrait dialogue véritablement avec le recueil précédent en reprenant ce thème essentiel de l'absence à soi-même ${ }^{3}$.

$$
+++
$$

1 J'emprunte ce mot à Dominique Rabaté qui l'emploie dans "Énonciation poétique, énonciation lyrique», Dominique Rabaté (dir.), Figures du sujet lyrique, Paris, Presses universitaires de France, coll. «Perspectives littéraires», 1996, p. 66. 2 Philippe More, Brouillons pour un siècle abstrait, Montréal, Poètes de brousse, 2008, 92 p. 3 J'ai abordé cette question à propos de Nos images d'aphasie dans ma chronique intitulée «Platitudes et densités de la poésie contemporaine», Voix et Images, vol. XXXIII, nº 3, printemps-été 2008, p. 124-128. 
Cette absence à soi n'est jamais simple négativité, et encore moins cynisme. Il serait malvenu de lire les titres de More (et partant, les recueils qu'ils coiffent) comme autant de postures de refus à partir desquelles le poète chercherait à s'abstraire du temps ou du monde. L'abstraction, l'aphasie et l'apesanteur sont tour à tour jouées chez lui comme autant de mécanismes (images ou ébauches au sein d'un thêatre du monde) permettant justement de retrouver le monde sensible, cela, au moment même où la perte des repères oblige le sujet à repenser ses «motifs pour l'amnésie» (24) ou l'inintelligibilité "de ce monde» qui ne fournit «aucune narration/à substituer aux circonstances» à celui qui «énumère [s]es leucémies » (25). Pour notre plus grand bonheur (bonheur esthétique, bien sûr), Philippe More n'en démord pas: la maladie, sous différentes formes, hante de part en part son recueil. Le coma, l'oxygène, l'ADN et tutti quanti peuplent ces poèmes où même les termes les plus techniques laissent surgir l'optimisme, comme dans «Une apoptose»:

\author{
et tu assisteras peut-être \\ à l'odeur des sargasses \\ qui accompagne de loin la mort enfouie \\ dans la blancheur des chromosomes \\ pendant que tu joueras \\ à te reconnaître parmi ce brouillon \\ un visage allumé de biais \\ par sa soudaine alchimie \\ de toutes les noyades. (43)
}

Le processus moléculaire d'autodestruction s'accompagne de la possibilité symbolique d'un renouvellement grâce au réseau sémantique de la naissance ${ }^{4}$ inscrit dans la première strophe. Mourir pour mieux renaître: ainsi dit, bien sûr, le tout semble banal, presque cliché. Ce serait toutefois mal rendre compte de la densité des Brouillons pour un siècle abstrait que de tenter de les réduire à cette seule formule, qui leur est pourtant essentielle dans la mesure où elle témoigne de ce refus du mondain partout présent dans la poésie de Philippe More.

J'entends ici le mondain au sens que lui donne Laurence Bougault quand, suivant là-dessus Georges Molinié, elle en parle comme de «l'ensemble des médiations entre le monde et l'homme ${ }^{5} »$. Pour Bougault, le réel (qui est pour elle un synonyme du monde) nous apparaît connaissable à cause du reflet que nous en offre le mondain. Autrement dit, parce que l'Homme est un être de langage, le mondain lui semble offrir un lieu de perception correct de la réalité du monde. Ainsi, l'auteure fait l'hypothèse que «le poète, conscient que le mondain n'est pas le monde, que la

$$
+++
$$

4 La sargasse est une algue dans laquelle vont pondre les anguilles; il me semble conséquemment pouvoir lire une allusion au liquide spermatique dans la blancheur des chromosomes. 5 Laurence Bougault, Poésie et réalité, Paris, L’Harmattan, coll. «Critiques littéraires», 2005, p. 13. 
langue constitue un système fermé sur soi, tente néanmoins de joindre le monde et de rétablir, dans la langue, un contact avec l'extra-textuel ${ }^{6}{ }$.

On aura beau dire, tous ceux qui écrivent des vers n'atteignent pas nécessairement au langage poétique, qui consiste à outrepasser ce mondain dont je parle ici. Or, s'il est un mérite qui revient à Brouillons pour un siècle abstrait, c'est très précisément de tâcher à accomplir cela. Chaque vers de Philippe More constitue un effort palpable pour sortir le sujet du mondain et lui imposer la relation langagière durable et nouvelle du "poème en différé» (56). L'accession au monde et à son intelligence provient de ce que le poète y fait entendre un langage déchargé de la perception usuelle des mots pour recouvrir une signification qui déborde inlassablement le mondain afin de "se frayer une mémoire/dans les traces victoriennes de l'ennui en surface $[\ldots] / / c^{\prime}$ est le principe syntaxique/de la moindre gerçure la musique aérée/ qui se transvase d'âme en âme» (50). Sans à proprement parler être une synesthésie, le transvasement ontologique est pourtant aussi chez More une transposition esthétique que la musique, à la fois omniprésente et obsédante, réalise sur les plans thématique et structurel.

La musique, qui «t'exagère en bégayant une à une/toutes les vies dont tu disposeras demain » (50), apparaît en effet comme un thème récurrent des soixantehuit poèmes du recueil, présente sous la forme d'un genre musical (le requiem), d'un tempo (l'adagio) ou par la nomination d'un compositeur, comme ce "Bach [qui] pleure dans toi» (42) et "s'entasse dans les replis d'une vie antérieure» (56). Le thème musical n'est pas ici d'ordre décoratif; toujours, la musique reste, dans ces poèmes, éprouvée depuis le lieu du sujet lyrique. Son tempo agit sur le récit du monde livré en prescrivant un temps distinctif qui institue conséquemment un ordre narratif particulier. Dans le poème "Adagio", le poète stipule donc «sous la musique avoir changé de fable/être arrivé après sa propre tragédie » (82). L'ordre mondain, on le voit, se trouve renversé, voire annihilé. Il s'agit de retrouver la préséance d'une vérité que seul le transvasement esthétique et musical semble capable d'opérer. Dans "Adagio deux», le poème suivant, il implore donc «tous les détails du paysage/à l'aube d'à moitié fleurir/comme une partition/qui essouffle ses images tièdes» (83).

Revenant au temps d'avant la parole mondaine, le poète laisse surgir la musicalité d'un Verbe qui, par la seule force de sa propagation, peut recréer le monde en fonction d'un ordre fusionnel où bat la mesure de son propre temps, justement peut-être parce que «nous n'avons rien appris hier/pas même à persister comme une musique infirme» (14). Comme une symphonie - ou un opéra - la musique offre la possibilité aux corps disloqués de retrouver une unité «hors de la minuterie» (20) mécanique de la machinerie clinique. Partant de la matérialité grotesque de la maladie, la poésie retrouve une modalité esthétique d'incarnation où le corps malade est aussi un corps sacré:

je revis la mort des autres comme un opéra

où le bruit s'accroche en forme de prière

$$
+++
$$

6 Laurence Bougault, ouvr. cité, p. 13. 
assez d'histoire pour cracher un évangile

en autant de langues qu'il faut pour se taire

en assez de versions tristement animales

pour suffire à la genèse la plus somnambule. (92)

Les «brouillons» de Philippe More sont donc, au sens fort, des ébauches plutôt que des poèmes inachevés, et l'abstraction du siècle n'y a pas valeur de fuite absconse hors du réel. Ces brouillons constituent autant de formes premières dont les imperfections, endossées, ouvrent la voie à la réformation esthétique d'une parole qui puisera dans son caractère idéel pour refaire le monde sensible. La musique y agit ainsi comme le lieu où il devient possible de se débarrasser des fausses images du «scénario [qui] ne suffit plus pour te cacher/du retard que tu ne sais plus prendre/sur ta propre vie» (88). Le tempo de la musique construit graduellement la marche (scénaristique) où le poète "pleure par sémaphores répétitions d'étoiles» (92).

S'agirait-il pour autant de faire le récit du deuil de la «petite sœur dans le cocon d'un autre siècle» (78)? Je n'en sais rien, et je refuse de toute façon de m'aventurer du côté de l'explication biographique pour expliquer le miracle poétique de ces vers qui disent la sacralité du corps médicalisé. Et s'il faut rappeler que Philippe More est médecin, je ne veux comprendre Brouillons pour un siècle abstrait que dans cette capacité du poète à dépasser le stade technique, scientifique, de son métier pour nous offrir ce qu'il en reste de plus fragilisant, déployant du coup le socle artistique depuis lequel ses mots esquissent le temps spirituel d'un recommencement, où le caractère sublime de la musique forme la trame de son éternel recentrement. Multiples sont les présences de l'absence en poésie québécoise mais, cette fois, la présence est celle à venir d'une absence actuelle alors que «ce que la mort imite sous les doigts c'est le monde/avec sa peau d'exil sur les contours de la mémoire» (92).

\author{
Le cri filé d'un trait \\ Tendu droit \\ D'une seule volée \\ À la pointe du cœur. \\ - Anne Hébert
}

À l'instar du cri hébertien placé en exergue, le ton adopté par Catherine Morency est plus dur et tranchant que celui de Philippe More, cela malgré le refus du mondain qui les rallie. La parole, dans Sans Ouranos ${ }^{7}$, écorche bien littéralement. Sa dureté n'est pas sans faire penser à celle - plus clinique, évidemment - d'une Sarah Kane cherchant à contrôler la perte irrémédiable dont elle se fait le témoin pressant et

$$
++
$$

7 Catherine Morency, Sans Ouranos, Québec, Le Lézard amoureux, 2008, 110 p. 
pressé. Nous sommes ici face à une parole féminine pleinement assumée, assurée de sa capacité à formuler l'informulable «d'une hygiène splendide/flamboyante incongrue/à laquelle/malgré la terrifiante/potentialité du ratage/ensemble/nous appartenons» (31).

Pour peu, on aurait envie de reprendre les mots de Pierre Emmanuel à propos de la poésie d'Anne Hébert quand il parle de «poèmes comme tracés dans l'os par la pointe d'un poignard ${ }^{8}$ ». Ainsi dégagé de la protection divine dès le titre de son recueil, le sujet lyrique de Catherine Morency replace en effet le corps au centre de l'univers qu'il déploie. Mais ce corps est à la fois meurtri et réduit à l'essentiel de la voix qui le constitue et le porte, ce qui indique l'espèce de refus constant de toute enflure caractérisant la déchirure profonde inscrite dans ses poèmes. "Sortir de la danse/laisser à Hérodiade/le spasme de ses hanches/puis casser d'un coup sec/le béotien calcul/des scribes sans échine.» (26)

Le projet est risqué, le pari est immense, presque insolent: il s'agit en somme de reconstruire la parole sans l'aide des dieux en vue d'atteindre le fondement de la conscience où cette parole se manifestera effectivement. De manière tout à fait habile, la disposition typographique des poèmes de Catherine Morency reprend cette idée d'un déploiement vertical et plongeant de la parole alors que bien souvent chaque mot du poème se trouve isolé des autres sur la ligne:

et
je
t'accueillerai
avec
tout
ce
dont
je
n'ai
pas
le
pouvoir. (93)

Simple jeu ou tic d'écriture? Il me semble voir dans cet agencement une mise à l'épreuve palpable de l'urgence singulière qui caractérise la voix de Sans Ouranos. Car enfin, en se donnant à lire verticalement, le cri poétique du second recueil de Catherine Morency refuse le réconfort éventuel d'une horizontalité représentant la mesure possible du point de fuite qui attendrait le sujet. La verticalité typographique des vers signale bien l'exigence radicale de la posture adoptée, toujours confrontée à l'impossibilité d'étaler son plein déploiement, constamment ramenée vers ellemême, dans un monde où la parole ne bénéficie plus de la protection du Ciel. De

$$
++
$$

8 Pierre Emmanuel, «Présentation», Anne Hébert, Le tombeau des rois, Québec, Institut littéraire du Québec, 1953, p. 7. 
cette façon, les mots deviennent saccadés; la syntaxe est constamment freinée par les hiatus du blanc devenu prépondérant. La construction paratactique des phrases maintient une cadence effrénée qui restitue à la face du lecteur la chute menaçante, «escalade/de/feux/sur/un/ciel/sans/crampons» (74).

Les luttes et les combats tout autant que l'effort fourni serviront à récupérer un espace qui semble avoir par trop longtemps été injustement investi. «[N]ous/ marcherons/blanchis/par une vieille/frondeuse», comme pour pouvoir enfin, dans les quatre derniers vers du recueil, «dénaturer/la fureur/par/la grâce» (111). Le caractère sublime de la parole se sera joué dans l'espace d'une fureur finalement surmontable justement parce que l'insurrection à laquelle elle a donné lieu aura favorablement servi la métamorphose de "ces furies/accrochées/à nos tempes» (9) du poème liminaire. Dans Sans Ouranos, tout se passe comme si la représentation avait assez duré. Le recueil exige le rétablissement immédiat de cette nécessité d'une parole poétique constamment en butte au caractère superflu du mondain en vue de réaliser le plein accomplissement d'une dialectique profonde dont sont exclus les repères réconfortants.

L'esthétisation du Verbe reste un enjeu majeur de Brouillons pour un siècle abstrait et Sans Ouranos. En y travaillant formellement leur propos comme ils l'ont fait, les deux poètes ont offert une poésie où le flegme de l'un et les injonctions de l'autre dévoilent le pouvoir du «[m]ot qui nourrit et comble comme le dévoilement soudain d'une verité ${ }^{9}$ » exigeante et brutale.

9 Roland Barthes, "Y a-t-il une écriture poétique?», Le degré zéro de l'écriture, Paris, Éditions du Seuil, coll. «Points. Littérature», 1972, p. 37. 